\title{
Rainbow Matchings in Properly Colored Bipartite Graphs
}

\author{
Guanghui Wang, Guizhen Liu \\ School of Mathematics, Shandong University, Jinan, China \\ Email: \{ghwang, gzliu\}@sdu.edu.cn
}

Received January 19, 2012; revised February 16, 2012; accepted March 25, 2012

\begin{abstract}
Let $G$ be a properly colored bipartite graph. A rainbow matching of $G$ is such a matching in which no two edges have the same color. Let $G$ be a properly colored bipartite graph with bipartition $(X, Y)$ and $\delta(G)=k \geq 3$. We show that if $\max \{|X|,|Y|\} \geq \frac{7 k}{4}$, then $G$ has a rainbow coloring of size at least $\left\lfloor\frac{3 k}{4}\right\rfloor$.
\end{abstract}

Keywords: Rainbow Matching; Bipartite Graphs

\section{Introduction and Notation}

We use [1] for terminology and notations not defined here and consider simple undirected graphs only. Let $G=(V, E)$ be a graph. A proper edge-coloring of $G$ is a function $c: E \rightarrow \mathbb{N}$ ( $\mathbb{N}$ is the set of non-negative integers) such that any two adjacent edges have distinct colors. If $G$ is assigned such a coloring $c$, then we say that $G$ is a properly edge-colored graph, or simply a properly colored graph. Let $c(e)$ denote the color of the edge $e \in E$. For a subgraph $H$ of $G$, let

$$
c(H)=\{c(e): e \in E(H)\} .
$$

A subgraph $H$ of $G$ is called rainbow if its edges have distinct colors. Recently rainbow subgraphs have received much attention, see the survey paper [2]. Here we are interested in rainbow matchings. The study of rainbow matchings began with the following conjectures.

Conjecture 1. (Ryser [3,4]) Every Latin square of odd order has a Latin transversal.

Conjecture 2. (Stein [5]) Every Latin square of order $n$ has a partial Latin transversal of size at least $n-1$.

An equivalent statement is that every proper $n$-edgecoloring of the complete bipartite graph $K_{n, n}$ contains a rainbow matching of size $n-1$; Moreover, if $n$ is odd, there exists a rainbow perfect matching. Hatami and Shor [6] proved that there is always a partial Latin transversal (rainbow matching) of size at least $n-O\left(\log ^{2} n\right)$.

Another topic related to rainbow matchings is orthogonal matchings of graphs. Let $G$ be a graph on $n$ vertices which is an edge disjoint union of $m k$-factors (i.e. $k$ regular spanning subgraphs). We ask if there is a matching $M$ of $m$ edges with exactly one edge from each $k$-factor? Such a matching is called orthogonal because of applications in design theory. A matching $M$ is suborthogonal if there is at most one edge from each $k$-factor. Alspach [7] posed the above problem in the case $k=2$. Stong [8] proved that if $n \geq 3 m-2$, then there is a such orthogonal matching. For $k=3$, the answer is yes, see [9]. In the same paper, Anstee and Caccetta proved the following theorem when $k=1$.

Theorem 3. [9] Let $G$ be an m-regular graph on $n$ vertices. Then for any decomposition of $E(G)$ into $m$ 1-factors $F_{1}, F_{2}, \cdots, F_{m}$, there is a matching $M$ of $p$ edges, at most one edge from each 1-factor, with

$$
p>\min \left\{\frac{n}{2}-\frac{3}{2}\left(\frac{n}{2}\right)^{\frac{2}{3}}, m-\frac{3}{2} m^{\frac{2}{3}}\right\} .
$$

In any decomposition of $E(G)$ into $m k$-factors, we can construct an edge colored graph by giving each $k$ factor a color. Then a rainbow matching of $G$ corresponds to a suborthogonal matching of $G$. In particular, when $k=1$, the edge colored graph obtained above is properly colored. So we can pose a more general problem: Let $G$ be a properly colored graph of minimum degree $\delta(G)$. Is there a rainbow matching of size $\delta(G)$ ? Unfortunately, the answer is negative, see [10]. Moreover, if $G$ is a properly colored complete graph, then $G$ has no rainbow matching of size more than $\left\lceil\frac{\delta(G)}{2}\right]$. In addition, the following theorem was shown in [11].

Theorem 4. [8] Let $G$ be a properly colored graph, $G \neq K_{4}$, and $|V(G)| \neq \delta(G)+2$. Then $G$ contains $a$ rainbow matching of size $\left\lceil\frac{\delta(G)}{2}\right\rceil$. 
However, we believe that if the order of a properly colored graph $G$ is much larger than its minimum degree $\delta(G)$, there should be a rainbow matching of size $\delta(G)$. In [10], we propose the following problem.

Problem 5. [10] Is there a function $f(n)$ such that for each properly colored graph $G$ with $|V(G)| \geq f(\delta(G))$, G must contain a rainbow matching of size $\delta(G)$ ?

Since when $n$ is even, an $n \times n$ Latin square has no Latin transversal (perfect rainbow matching) (see [3]), if the function $f(n)$ exists, $f(n)$ should be greater than $2 n$. Motivated by this problem, we prove the following results in [10].

Theorem 6. [10] Let $G$ be a properly colored graph and $|V(G)| \geq \frac{8 \delta(G)}{5}$. Then $G$ has a rainbow matching of size at least $\left\lfloor\frac{3 \delta(G)}{5}\right\rfloor$.

Theorem 7. [10] Let $G$ be a properly colored trianglefree graph. Then $G$ has a rainbow matching of size at least $\left\lfloor\frac{2 \delta(G)}{3}\right\rfloor$.

In [12], Wang, Zhang and Liu proved that if

$$
f(n)>\frac{n^{2}+14 n+1}{4},
$$

then $G$ has a rainbow matching of size $\delta$, which answers the above question in the affirmative. Eiemunsch et al. [13] improved this bound to $\left\lfloor\frac{13 n}{2}-\frac{23}{2}+\frac{41}{8 n}\right\rfloor+1$. Later, this bound was improved to $\frac{9 n-5}{2}$ by Lo in [14]. In this paper, we consider the rainbow matching of the properly colored bipartite graph, and prove the following result.

Theorem 8. Let $G$ be a properly colored bipartite graph with bipartition $(X, Y)$ and $\delta(G)=k \geq 3$. If $\max \{|X|,|Y|\} \geq \frac{7 k}{4}$, then $G$ has a rainbow coloring of size at least $\left\lfloor\frac{3 k}{4}\right\rfloor$.

For more result about rainbow matchings under the color degree conditions, we refer to $[15,16]$.

\section{Proof of Theorem 8}

Let $G=(X, Y)$. Without loss of generality, we assume that $\max \{|X|,|Y|\}=|Y|$. Suppose that our conclusion is not true, we choose a maximum rainbow matching $M$. Let $t=|M|$. Without loss of generality, we assume that

$$
c(M)=\{1,2, \cdots, t\} \text {. }
$$

Then $t \leq\left\lfloor\frac{3 k}{4}\right\rfloor-1$. Let $X \cap V(M)=X_{1}$ and $Y \cap V(M)=Y_{1}$.

Put $Y-Y_{1}=Y_{2}$ and $X-X_{1}=X_{2}$. Let $X_{11}$ denote the vertices in $X$ which are incident with $Y_{2}$ by three edges with new colors. Clearly, $X_{11} \subseteq X_{1}$. Otherwise, we can get a rainbow matching of size at least $t+1$, which is a contradiction. Let $Y_{11}$ denote the vertices which are incident with the vertices in $X_{11}$ by the edges in $M$. We have the following claim.

Claim 1. $\left|X_{11}\right| \geq\left\lceil\frac{k}{4}\right\rceil$.

Proof. Let $y_{i} \in Y_{2}$. If there is an edge $x y_{i}$ such that $c\left(x y_{i}\right) \notin c(M)$, then $x \in X_{1}$. Otherwise, there is a rainbow matching $M \cup x y_{i}$ of size $t+1$, which is a contradiction. Let $E_{1}$ denote the edges which are incident with vertices in $Y_{2}$ and have new colors. Since each vertex in $Y_{2}$ has degree at least $k$,

$$
\left|E_{1}\right| \geq(k-t)\left|Y_{2}\right| \geq\left(\left\lceil\frac{k}{4}\right\rceil+1\right)\left|Y_{2}\right| \text {. }
$$

On the other hand, $\left|E_{1}\right| \leq\left|Y_{2}\right|\left|X_{11}\right|+2\left(\left|X_{1}\right|-\left|X_{11}\right|\right)$. So we have the following equality

$$
\left(\left\lceil\frac{k}{4}\right\rceil+1\right)\left|Y_{2}\right| \leq\left|Y_{2}\right|\left|X_{11}\right|+2\left(\left|X_{1}\right|-\left|X_{11}\right|\right) \text {. }
$$

Hence

$$
\begin{aligned}
\left|X_{11}\right| & \geq \frac{\left(\left\lceil\frac{k}{4}\right\rceil+1\right)\left|Y_{2}\right|-2\left|X_{1}\right|}{\left|Y_{2}\right|-2} \\
& \geq \frac{\left.\left(\left\lceil\frac{k}{4}\right\rceil+1\right)\left|Y_{2}\right|-2\left(\mid \frac{3 k}{4}\right\rfloor-1\right)}{\left|Y_{2}\right|-2} \\
& =\frac{\left(\left\lceil\frac{k}{4}\right\rceil+1\right)\left(\left|Y_{2}\right|-2\right)-2(k-2)}{\left|Y_{2}\right|-2} .
\end{aligned}
$$

Since $|Y| \geq \frac{7 k}{4},\left|Y_{2}\right| \geq \frac{7 k}{4}-\left(\left\lfloor\frac{3 k}{4}\right\rfloor-1\right) \geq k+1$, thus $\left|X_{11}\right| \geq\left\lceil\frac{k}{4}\right\rceil$.

Without loss of generality, we assume that

$$
X_{11}=\left\{x_{1}, x_{2}, \cdots, x_{p}\right\},
$$

where $p \geq\left\lceil\frac{k}{4}\right\rceil$. Let $y_{i}$ denote the vertex which is incident with $x_{i}$ by an edge in $M$.

Claim 2. Let $x_{i}$ be any vertex in $X_{11}$ and $y_{i}$ denote the vertex which is incident with $x_{i}$ by an edge in $M$. If $y_{i}$ is incident with a vertex $x \in X_{2}$, then 
$c\left(x y_{i}\right) \in\{p+1, \cdots, t\}$.

Proof. Suppose our conclusion does not hold. First, we know that $c\left(x y_{i}\right)$ can not be a new color. Otherwise, since $x_{i}$ are incident with three edges having new colors, we can choose one edge (say $e$ ). Then we can get a new rainbow matching of size $t+1$ by adding $x y_{i}, e$ and deleting $x_{i} y_{i}$. Thus we will get a contradiction. So we conclude that $c\left(x y_{i}\right) \in\{1,2, \cdots, p\}$. Since $G$ is properly colored, $c\left(x y_{i}\right) \neq i$. So without loss of generality, we assume that $c\left(x y_{i}\right)=1$. Moreover, we assume that the edges with new colors are incident with $x_{1}$ are $e_{1}$, $e_{2}$ and $e_{3}$. Now we can choose $e_{j}(j=1,2,3)$ such that $c\left(e_{j}\right) \neq c(e)$ and $e_{j}$ is not incident with $y$. Hence we have a new rainbow matching by adding $e_{j}, x y_{i}, e$ and deleting $x_{i} y_{i}, x_{1} y_{1}$, which is a contradiction.

Claim 3. If there exists an edge $x y$ such that $x \in X_{2}$ and $y \in Y_{2}$, then $c(x y) \in\{p+1, \cdots, t\}$.

Proof. Suppose, to the contrary, $c(x y) \notin\{p+1, \cdots, t\}$. If $c(x y)$ is a new color, then clearly there exists a rainbow matching $M \cup x y$ of size $t+1$. So we assume that $c(x y) \in\{1,2, \cdots, p\}$. Without loss of generality, we assume that $c(x y)=1$. We can also choose one edge $e$ incident with $x_{1}$ such that $e$ is not incident with $y$ and $c(e)$ is a new color. Then we can also obtain a rainbow matching by adding $e, x y$ and deleting $x_{1} y_{1}$, which is a contradiction. This completes the proof of Claim 3.

Let $x y$ be an edge such that $x \in X_{2}$ and $y \in Y_{11} \cup Y_{2}$. By Claim 2 and Claim 3, $c(x y) \in\{p+1, \cdots, t\}$. Let $X_{12}=X_{1}-X_{11}$ and $Y_{12}=Y-Y_{11}$. Since $d(x) \geq k$,

$$
\left|X_{12}\right|=\left|Y_{12}\right| \geq k-(t-p) \text {. }
$$

On the other hand, $\left|X_{12}\right|=\left|X_{1}\right|-\left|X_{11}\right| \leq t-p$. Hence $t-p \geq k-(t-p)$. That is $t \geq \frac{k+2 p}{2} \geq\left\lfloor\frac{3 k}{4}\right\rfloor$, which contradicts with $t \leq\left\lfloor\frac{3 k}{4}\right\rfloor-1$. This completes the whole proof.

\section{Acknowledgements}

We would like to thank the referee for the careful review and the valuable comments. This research was supported by NSFC Grants (61070230, 11101243), IIFSDU (2009 hw001), RFDP (20100131120017) and SRF for ROCS.

\section{REFERENCES}

[1] J. A. Bondy and U. S. R. Murty, "Graph Theory with
Applications,” Macmillan Press, New York, 1976.

[2] M. Kano and X. Li, "Monochromatic and Heterochromatic Subgraphs in Edge-Colored Graphsa Survey," Graphs and Combinatorics, Vol. 24, No. 4, 2008, pp. 237-263. doi:10.1007/s00373-008-0789-5

[3] R. A. Brualdi and H. J. Ryser, "Combinatorial Matrix Theory,” Cambridge University Press, Cambridge, 1991.

[4] H. J. Ryser, "Neuere Probleme der Kombinatorik," in Vortrage uber Kombinatorik Ober-Wolfach, Mathematisches Forschungsinstitut Oberwolfach, 1967, pp. 24-29.

[5] S. K. Stein, "Transversals of Latin Squares and Their Generalizations,” Pacific Journal of Mathematics, Vol. 59, No. 2, 1975, pp. 567-575.

[6] P. Hatami and P. W. Shor, "A Lower Bound for the Length of a Partial Transversal in a Latin Square,” Journal of Combinatorial Theory, Series A, Vol. 115, No. 7, 2008, pp. 1103-1113. doi:10.1016/j.jcta.2008.01.002

[7] B. Alspach, "Problem 89," Discrete Mathematics, Vol. 69, 1988, p. 106.

[8] R. Stong, “Orthogonal Matchings,” Discrete Mathematics, Vol. 256, No. 1-2, 2002, pp. 515-518. doi:10.1016/S0012-365X(01)00315-6

[9] R. P. Anstee and L. Caccetta, "Orthogonal Matchings," Discrete Mathematics, Vol. 179, No. 1-3, 1998, pp. 37-47. doi:10.1016/S0012-365X(97)00025-3

[10] G. Wang, "Rainbow Matchings in Properly Edge Colored Graphs,” The Electronic Journal of Combinatorics, Vol. 18, 2011, Paper \#P162.

[11] T. D. LeSaulnier, C. Stocker, P. S. Wenger and D. B. West, "Rainbow Matching in Edge-Colored Graphs," The Electronic Journal of Combinatorics, Vol. 17, 2010, Paper \#N26.

[12] G. Wang, J. Zhang and G. Liu, "Existence of Rainbow Matchings in Properly Edge-Colored Graphs,” Frontiers of Mathematics in China. doi:10.1007/s11464-012-0202-9

[13] J. Diemunsch, M. Ferrara, C. Moffatt, F. Pfender and P. S. Wenger, "Rainbow Matchings of Size $\delta(G)$ in Properly Edge-Colored Graphs,” Arxiv Preprint, arXiv: 1108. 2521, 2011.

[14] A. Lo, “A Note on Rainbow Matchings in Properly EdgeColoured Graphs,” Arxiv preprint, arXiv:1108.5273v1, 2011.

[15] H. Li and G. Wang, "Color Degree and Heterochromatic Matchings in Edge-Colored Bipartite Graphs,” Utilitas Mathematica, Vol. 77, 2008, pp. 145-154.

[16] G. Wang and H. Li, "Heterochromatic Matchings in EdgeColored Graphs,” Electronic Journal of Combinatorics, Vol. 15, 2008, Paper \#R138. 